\title{
THE
}

1976

\section{Percus-Yevick and Hypernetted-Chain Approximations}

\author{
A. Choudry \\ University of Rhode Island \\ Pranab K. Banerjee \\ University of Rhode Island, pbanerjee@uri.edu \\ Narinder K. Allawadi
}

Follow this and additional works at: https://digitalcommons.uri.edu/phys_facpubs

Terms of Use

All rights reserved under copyright.

\section{Citation/Publisher Attribution}

Choudry, A., Banerjee, P. K., \& Ailawadi, N. K.. (1976). Percus-Yevick and hypernetted-chain approximations. Physical Review A, 14(1), 434-437. doi: 10.1103/PhysRevA.14.434

Available at: http://dx.doi.org/10.1103/PhysRevA.14.434

This Article is brought to you for free and open access by the Physics at DigitalCommons@URI. It has been accepted for inclusion in Physics Faculty Publications by an authorized administrator of DigitalCommons@URI. For more information, please contact digitalcommons-group@uri.edu. 


\title{
Percus-Yevick and hypernetted-chain approximations*
}

\author{
A. Choudry \\ Department of Physics, University of Rhode Island, Kingston, Rhode Island 02881 \\ P. K. Banerjee \\ Department of Physics, Rhode Island Junior College, Warwick, Rhode Island 02886
}

Narinder K. Ailawadi

Institut für Theoretische Physik (Kondensierte Materie), Freie Universität Berlin, West Berlin, Germany

(Received 10 November 1975)

\begin{abstract}
Percus-Yevick and hypernetted-chain models connecting the structure factor $S(k)$ with effective pair potential $\phi(r)$ have been studied in detail. Recent neutron-scattering data of Yarnell et al., for liquid argon at $85^{\circ} \mathrm{K}$, have been employed to generate these potentials. Using 0.01 uncertainty in the experimental data, the uncertainty in the pair potentials has been computed. Having developed a general method of error propagation analysis, the effect of experimental errors in $S(k)$ on Percus-Yevick and hypernetted-chain potentials is investigated and the resulting potentials are compared with Lennard-Jones and Barker, Fisher, and Watts potentials.
\end{abstract}

\section{INTRODUCTION}

Recent accurate measurements of structure factors $S(k)$ have led to a renewed interest in the approximate theories of liquids. Typically, the data for the measured structure factor are inserted in one or the other of integral equations used in the theory of liquids, and information about the pair potentials is obtained. This procedure has been used for example, for liquid metals, particularly liquid $\mathrm{Na}$ and $\mathrm{K},{ }^{1-6}$ in order to calculate effective pair potentials.

More recently, Yarnell et al. ${ }^{7}$ have measured structure factor of liquid argon at $85^{\circ} \mathrm{K}$ with an accuracy of 0.01 by neutron-diffraction techniques. These experimental data are in agreement with the molecular-dynamics data on $S(k)$ of Verlet, ${ }^{8}$ based on the Lennard-Jones (L-J) potential (6-12),

$$
\phi(r)=4 \epsilon\left[(\sigma / r)^{12}-(\sigma / r)^{6}\right]
$$

with $\epsilon / k_{B}=119.8^{\circ} \mathrm{K}$ and $\sigma=3.405 \AA$ as parameters.

The data of Yarnell et al. ${ }^{7}$ are also in good agreement with the simulations based on a rather different pair potential, which includes three-body effects. ${ }^{9}$ Thus, one is led to the conclusion ${ }^{7}$ that liquid argon can be equally well described by the L-J potential, Eq. (1), or by the Barker, Fisher, and Watts (BFW) potential. ${ }^{9}$

In an effort to obtain some information about the effective pair interaction, the Percus-Yevick (PY) and hypernetted-chain (HNC) approximations have been studied for a long time. ${ }^{10}$ It has been pointed out $^{8,11}$ that the PY equation (and perhaps HNC) leads to quite accurate pair potentials at low densities but breaks down at liquid densities. Also, in order to obtain $10 \%$ accuracy in the calculated pair potentials, an accuracy of $1 \%$ is needed in the measured structure factor.$^{8,11,12}$ More recently, it has been shown ${ }^{13}$ that the equilibrium structure of simple liquids is dominated by the repulsive part of the potential and that the attractive part of the potential can be treated as a perturbation. ${ }^{14}$

In this paper we reconsider the following problem: Given the equilibrium structure factor, can we obtain information about the pair potential? In particular, we restudy the PY and HNC equations for the reason that more accurate data on $S(k)$ are now available, and thus the limits of the reliability of the inversion process need to be thoroughly investigated; i.e., for a given accuracy in the structure-factor measurements, how much error is propagated in computing $\phi_{\mathrm{PY}}(r)$ and $\phi_{\mathrm{HNC}}(r)$, the potentials obtained from PY and HNC equations; and we examine if their differences from L-J and BFW are due to experimental errors. Such a comparison might indicate if either of the two (PY and HNC) is closer to L-J. On theoretical grounds, there seems to be no clear argument for preferring one approximation or the other; the same is true for the Born-Green equation as well, although for liquid metals, the potential derived from Born-Green theory is usually preferred because of long-range Friedel oscillations.

For this purpose, a detailed analysis of the effect of the uncertainties of the input structurefactor data on the derived pair potentials $\phi_{\mathrm{PY}}(r)$ and $\phi_{\mathrm{HNC}}(f)$ is carried out. In particular, we have attempted to develop a general method for treating the errors, which yields not just the magnitude 
of the error in $\phi$ but the density distribution thereof in the $r$ - $\phi$ space.

\section{METHOD}

Experimentally one counts the number of neutrons scattered at an angle $\theta$ to determine the differential cross section $\sigma(\theta):{ }^{16}$

$$
\frac{d \sigma}{d \Omega}=\sigma(\theta)=\left\langle f(\theta)^{*} f(\theta)\right\rangle
$$

For $n$ scattering by a nucleus of mass $M$, atomic number $A, f(\theta)$ is given by ${ }^{15}[m=M /(A+)]$

$$
f(\theta)=-\frac{m}{2 \pi h^{2}} \int e^{i \overrightarrow{\mathrm{k}} \cdot \overrightarrow{\mathrm{r}}} V(\overrightarrow{\mathrm{r}}) d \overrightarrow{\mathrm{r}} .
$$

The potential $V(r)$ is assumed to be of Fermi type and is obtained by introducing a coherent scattering length $b$ and summing over all the $N$ particles:

$$
V(r)=\frac{2 \pi h^{2}}{m} \frac{b}{N} \sum_{i=1}^{N} \delta\left(\overrightarrow{\mathrm{r}}-\overrightarrow{\mathrm{r}}_{i}\right),
$$

which yields

$$
\sigma(\theta)=\frac{b^{2}}{N} \int e^{i \overrightarrow{\mathrm{k}} \cdot \overrightarrow{\mathrm{r}}}\left\langle\sum_{i j} \delta\left(\overrightarrow{\mathrm{r}}+\overrightarrow{\mathrm{r}}_{i}-\overrightarrow{\mathrm{r}}_{j}\right)\right\rangle d \overrightarrow{\mathrm{r}} .
$$

The pair distribution function $g(r)$ is defined by

$$
g(r)=\frac{1}{\rho N}\left\langle\sum_{i \neq j} \delta\left(\overrightarrow{\mathrm{r}}+\overrightarrow{\mathrm{r}}_{i}-\overrightarrow{\mathrm{r}}_{j}\right)\right\rangle
$$

so that

$$
d n(r)=n 4 \pi r^{2} g(r) d r,
$$

where $d n(r)$ is the number of particles in a spherical shell of radius $r$ and thickness $d r, n$ being the number of particles in a unit volume. Combining it with Eq. (5), we have

$$
\sigma(\theta)=b^{2}\left(\int \rho[g(r)-1] e^{i \overrightarrow{\mathrm{k}} \cdot \overrightarrow{\mathrm{r}}} d \overrightarrow{\mathrm{r}}+1+\rho \delta(k)\right)
$$

from which one defines the structure factor $S(k)$ :

$$
S(\overrightarrow{\mathrm{k}})=1+\rho \int e^{i \overrightarrow{\mathrm{k}} \cdot \overrightarrow{\mathrm{r}}}[g(r)-1] d \overrightarrow{\mathrm{r}} .
$$

By combining Eqs. (8) and (9), a simple relation between $\sigma(\theta)$ and $S(k)$ can be obtained. After integrating the angular part, $S(k)$ and $g(r)$ can be written as a pair of Fourier transforms:

$$
\begin{aligned}
& S(k)=1+\frac{4 \pi n}{k} \int[g(r)-1] r \sin k r d r, \\
& g(r)=1+A \int[S(k)-1] k \sin k r d k,
\end{aligned}
$$

with $A=1 /\left(2 \pi n^{2} r\right)$. This series of well-known steps Eqs. (2)-(11) is reviewed here to point out the sources and analysis of errors involved in this procedure and the subsequent calculation of the potentials. To convert the experimental neutron counts into $\sigma(\theta)$, a number of experimental parameters, such as cell geometry, detector efficiency, etc. are needed and all of these have some errors in them. To calculate $S(k)$ from $\sigma(\theta)$, cross sections and limiting values of $S(k \rightarrow 0)$ and $g(r-0)$ are needed. Details of the error analysis in this procedure are discussed by Yarnell et al. ${ }^{7}$ Symbolically the entire above analysis can be thought of as a transformation $T_{s \sigma}$ such that

$$
\rho_{s}(k)=T_{s \sigma} \rho_{\sigma}(\theta),
$$

where $\rho_{\sigma}(\theta)$ is a density distribution function in the $\theta-\sigma$ plane and is identical with the scattergram of the experimental points in that plane; $\rho_{s}(k)$ is the corresponding density distribution function in $k-s$ plane. Thus the fact that all experimental values of $S(k)$ are normally distributed about the mean $S_{0}(k)$ with a constant variance $v^{2}$ (e.g., $v=0.01$ ) can be stated as

$$
E\{S(k)\}=S_{0}(k)=\left.\int_{0}^{\infty} S(k) N\left(S_{0}, v^{2}\right) d S\right|_{k}
$$

and

$$
E\left\{\left(S-S_{0}\right)^{2}\right\}=v^{2},
$$

where $N\left(S_{0}, v^{2}\right)$ is a normal distribution with mean $S_{0}$ and variance $v^{2}$, and $E\{x\}$ is the expected value of $x$. Knowing $S(k)$ and $g(r)$ [from Eq. (11)] we can calculate $g(r)$ by using Eq. (11) and then the PY and HNC potentials can be calculated by the wellknown relations

$$
\begin{aligned}
& \phi_{\mathrm{PY}}=k_{B} T \ln [1-c(r) / g(r)], \\
& \phi_{\mathrm{HNC}}=k_{B} T[g(r)-c(r)-1-\ln g(r)],
\end{aligned}
$$

where

$$
c(r)=A \int_{0}^{\infty}[S(k)-1] \frac{k \sin k r}{S(k)} d k .
$$

We shall now discuss how the errors in $S(k)$ are propagated through $g(r)$ into $\phi_{\mathrm{PY}}$ and $\phi_{\mathrm{HNC}}$. The fact that the precision in calculating the pair potentials is strongly dependent on the measurement precision in $S(k)$, has been well established, e.g., Levesque and Verlet. ${ }^{11}$ Gehlen and Enderby ${ }^{17}$ developed a model of linear propagation of errors and applied it to $\mathrm{Na}$. However this model is rather restrictive and may lead to doubtful estimates. We shall now formulate the general problem and indicate a solution. Mathematically speaking, the experimental function $S(k)$ is a set of stochastic variables with arbitrary distributions in $k-S$ space and thus all operations on $S(k)$, e.g., differentiation, integration, etc, should be done in conformity with the rules appropriate to stochastic variables. We introduce an experimental error func- 
tion $\epsilon(k, S)$ :

$$
S(k)=S_{0}+\epsilon(k, S)
$$

so that

$$
E\{\epsilon(k, S)\}=0 \text {. }
$$

From Eq. (11) it can be shown that the error function $\Delta g(r)$ for $g(r)$ is given by the following stochastic integral:

$$
\Delta g(r)=A \int_{0}^{\infty} \epsilon(k, S) k \sin k r d k
$$

It should be noted that $\Delta g(r)$ is also a set of stochastic variables and Eq. (20) may be interpreted as a transformation $T_{g s}$, in the spirit of Eq. (12), i.e.,

$$
\Delta g(r)=T_{g s} \in(S, k) .
$$

Similarly using Eqs. (15), (16), and (17) the following can be defined:

$$
\begin{aligned}
& \Delta c(r)=T_{c s} \epsilon(S, k), \\
& \Delta \phi_{\mathrm{PY}}=T_{\mathrm{PS}} \epsilon(S, k), \\
& \Delta \phi_{\mathrm{HNC}}=T_{\mathrm{HS}} \epsilon(S, k) .
\end{aligned}
$$

All of the above involve stochastic integrals of the type in Eq. (20). Ballentine and Jones ${ }^{12}$ have replaced the above stochastic operations by a linear relation in assuming that the $T$ 's are matrices. As an illustration we shall indicate the evaluation of $\Delta g(r)$ from Eq. (20). For a finite number $n$ of experimental points, in machine computation,

Eq. (20) can be written as

$$
\Delta g(r)=\sum_{i=1}^{n} \epsilon_{i} k_{i} \sin k_{i} r \Delta k_{i},
$$

which is a sum of $n$ independent stochastic vari-

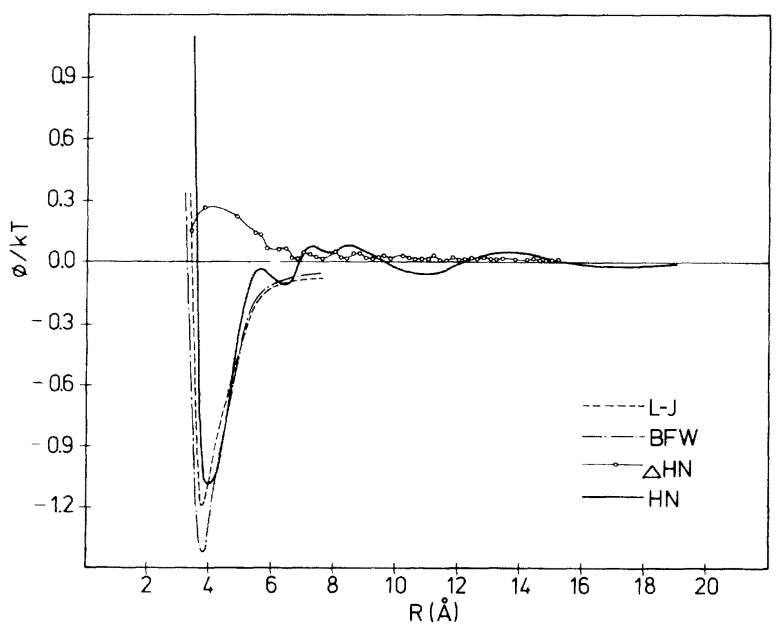

FIG. 1. $\Delta \phi_{\mathrm{HNC}}$ and $\phi_{\mathrm{HNC}}$ compared with L-J and BFW. ables $\eta_{i}$ where

$$
\eta_{i}=\epsilon_{i} k_{i} \sin k_{i} r \Delta k_{i} .
$$

The moment-generating function $\xi_{i}(t)$ of $\eta_{i}$ is defined as

$$
\xi_{i}(t)=\int_{0}^{\infty} \eta_{i}(s) e^{t s} d s
$$

in terms of which (25) can be written as

$$
\Delta g(r)=\int_{0}^{\infty}\left\{\prod_{i=1}^{n} \xi_{i}(t)\right\} e^{-g t} d t,
$$

which is a density distribution in $r-g$ space. Expressions to be similarly evaluated corresponding to Eqs. (22), (23), and (24) are

$$
\begin{aligned}
& \Delta c(r)=A \sum_{n=1}^{\infty}\left(\frac{-\epsilon(k)}{S_{0}}\right)^{n} \frac{k \sin k r}{S_{0}(k)} d k \\
& \Delta \phi_{\mathrm{HNC}}=\Delta g(r)+\Delta c(r)-\ln (1+\alpha) \\
& \Delta \phi_{\mathrm{PY}}=k_{B} T \ln \left[1-\frac{c}{g}\left(\alpha+(1+\alpha) \sum_{n=1}^{\infty}(-\beta)^{n}\right) e^{-\phi / k T}\right]
\end{aligned}
$$

where $\alpha=\Delta g / g$ and $\beta=\Delta c / c$. Thus in principle it is possible to translate the density of experimental points in the $k-s$ space into the corresponding density in $r-\phi$ space for arbitrary error functions.

\section{CONCLUSION}

Using the structure factor data of Ref. 7 and the error of 0.01 , we have calculated HNC and PY potentials and the errors in them. These potentials are compared with L-J and BFW in Figs. 1 and 2 , respectively (N.B. the errors in $\mathrm{HNC}$ and $\mathrm{PY}$ as displayed in these figures are magnified 10 times). The errors in PY (cf. Fig. 2) are much

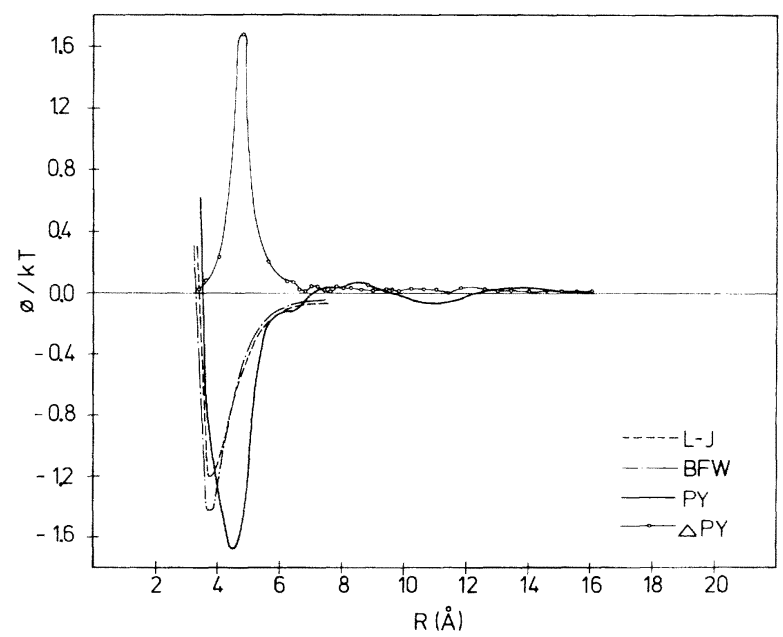

FIG. 2. $\Delta \phi_{\mathrm{PY}}$ and $\phi_{\mathrm{PY}}$ compared with L-J and BFW. 
more pronounced (up to $15 \%$ ) than in HNC (cf. Fig. 1). In particular, the well depth of PY is rather severely affected by the errors in $S(k) 0^{8,11,12}$ It is interesting to note that the well depth of HNC agrees with L-J better than with BFW. Of course this agreement does not necessarily imply the superior validity of one or the other potential. However, one can conclude that by using the general analysis developed here, the differences from L-J and BFW are beyond experimental errors. A recent perturbation calculation of Madden and Fitts $^{18}$ for liquid Ar based on HNC gives much more accurate results, and our analysis supports this conclusion.

\section{ACKNOWLEDGMENTS}

We would like to thank the staff of the URI computer center for the generous use of their facilities. N. K. A. would like to thank Professor A. Rahman for discussions and his interest in this problem; A. C. would like to express his gratitude to Professor Vernier for extending kind hospitality at Faculté des Science, Université de Dijon.
*Supported in part by the Deutsche Forschungs Gemeinschaft.

${ }^{1}$ Y. Was eda and K. Suzuki, in Properties of Liquid Metals, edited by S. Takeuchi (Taylor and Francis, London, 1973), p. 37.

${ }^{2}$ Y. Waseda and K. Suzuki, Phys. Stat. Sol. B $\underline{57}, 351$ (1973).

${ }^{3}$ W. S. Howells and J. E. Enderby, J. Phys. C $\underline{5}, 1277$ (1972).

${ }^{4}$ W. S. Howells, in Ref. 1, p. 43.

${ }^{5}$ N. K. Ailawadi, P. K. Banerjee, and A. Choudry, J. Chem. Phys. 60, 2571 (1974); 60, 5124 (1974).

${ }^{6}$ R. Kumaravadivel, R. Evans, and D. A. Greenwood, J. Phys. F $\underline{4}, 1839$ (1974).

${ }^{7}$ J. L.Yarnell, M. J. Katz, R. G. Wenzel, and S. K. Koenig, Phys. Rev. A 7, 2130 (1973).

${ }^{8}$ L. Verlet, Phys. Rev. $\underline{165}, 201$ (1967).

${ }^{9} \mathrm{~J}$. A. Barker, R. A. Fisher, and R. O. Watts, Mol. Phys. 21, 657 (1971).

${ }^{10} \mathrm{See}$, for example, P. G. Mikolaj and C. J. Pings, J.
Chem. Phys. 46, 1401 (1967); H. Fredrikze, C. D. Andriesse, and E. Legrand, Physica 62, 474 (1972).

${ }^{11}$ D. Levesque and L. Verlet, Phys. Rev. Lett. 20, 905 (1968).

${ }^{12}$ L. E. Ballentine and J. C. Jones, in Ref. 1, p. 51.

${ }^{13}$ D. Chandler and J. D. Weeks, Phys. Rev. Lett. 25, 149 (1970).

${ }^{14}$ H. C. Andersen, J. D. Weeks, and D. Chandler, Phys. Rev. A 4, 1597 (1971); H. C. Andersen, D. Chandler, and J. D. Weeks, J. Chem. Phys. 56, 3812 (1972).

${ }^{15}$ See, for example, P. A. Egelstaff, An Introduction to the Liquid State (Academic, London, 1967).

${ }^{16}$ W. A. Schlup, Symposium on Inelastic Scattering of Neutrons by Condensed Systems, Brookhaven National Laboratory Report No. BNL 940, 1965 (unpublished).

${ }^{17}$ P. C. Gehlen and J. E. Enderby, J. Chem. Phys. 51, 547 (1969)

${ }^{18}$ W. G. Madden and D. D. Fitts, J. Chem. Phys. $\underline{61}$, 5475 (1974); Mol. Phys. 30, 1809 (1975). 